\title{
Hypersensitivity Pneumonitis
}

\section{Radiologic Phenotypes Are Associated With Distinct Survival Time and Pulmonary Function Trajectory}

\author{
Margaret L. Salisbury, MD; Tian Gu, MS; Susan Murray, ScD; Barry H. Gross, MD; Aamer Chughtai, MBBS; \\ Mohamed Sayyouh, MBBCh; Ella A. Kazerooni, MD; Jeffrey L. Myers, MD; Amir Lagstein, MD; Kristine E. Konopka, MD; \\ Elizabeth A. Belloli, MD; Jamie S. Sheth, MD; Eric S. White, MD; Colin Holtze, MD; Fernando J. Martinez, MD; \\ and Kevin R. Flaherty, MD
}

BACKGROUND: Hypersensitivity pneumonitis (HP) is an interstitial lung disease with a better prognosis, on average, than idiopathic pulmonary fibrosis (IPF). We compare survival time and pulmonary function trajectory in patients with HP and IPF by radiologic phenotype.

METHODS: HP ( $\mathrm{n}=117$ ) was diagnosed if surgical/transbronchial lung biopsy, BAL, and exposure history results suggested this diagnosis. IPF $(\mathrm{n}=152)$ was clinically and histopathologically diagnosed. All participants had a baseline high-resolution CT (HRCT) scan and FVC \% predicted. Three thoracic radiologists documented radiologic features. Survival time is from HRCT scan to death or lung transplant. Cox proportional hazards models identify variables associated with survival time. Linear mixed models compare post-HRCT scan FVC \% predicted trajectories.

RESULTS: Subjects were grouped by clinical diagnosis and three mutually exclusive radiologic phenotypes: honeycomb present, non-honeycomb fibrosis (traction bronchiectasis and reticulation) present, and nonfibrotic. Nonfibrotic HP had the longest event-free median survival ( $>14.73$ years) and improving FVC \% predicted (1.92\%; 95\% CI, 0.49-3.35; $P=$ .009). HP with non-honeycomb fibrosis had longer survival than IPF (> 7.95 vs 5.20 years), and both groups experienced a significant decline in FVC \% predicted. Subjects with HP and IPF with honeycombing had poor survival (2.76 and 2.81 years, respectively) and significant decline in FVC \% predicted.

CONCLUSIONS: Three prognostically distinct, radiologically defined phenotypes are identified among patients with HP. The importance of pursuing a specific diagnosis (eg, HP vs IPF) among patients with non-honeycomb fibrosis is highlighted. When radiologic honeycombing is present, invasive diagnostic testing directed at determining the diagnosis may be of limited value given a uniformly poor prognosis.

CHEST 2019; 155(4):699-711

KEY WORDS: interstitial lung disease; prognostic model; pulmonary fibrosis

FOR EDITORIAL COMMENT, SEE PAGE 655

ABBREVIATIONS: $\mathrm{HP}=$ hypersensitivity pneumonitis; $\mathrm{HR}=$ hazard ratio; $\mathrm{HRCT}=$ high-resolution $\mathrm{CT}$; $\mathrm{ILD}=$ interstitial lung disease; IPF = idiopathic pulmonary fibrosis; LRT = likelihood ratio test; UIP = usual interstitial pneumonia
AfFiliations: From the Division of Pulmonary and Critical Care Medicine (Drs Salisbury, Belloli, Sheth, White, Holtze, and Flaherty) and the Departments of Biostatistics (Ms Gu and Dr Murray), Radiology (Drs Gross, Kazerooni, Chughtai, and Sayyouh), and Pathology (Drs Myers, Lagstein, and Konopka), University of Michigan, 
Hypersensitivity pneumonitis (HP) is a heterogeneous interstitial lung disease (ILD) caused by inhalational exposure to a variety of antigens. ${ }^{1}$ Patients with $\mathrm{HP}$ and radiologic or histopathologic evidence of pulmonary fibrosis experience shortened survival compared with those without pulmonary fibrosis. ${ }^{1-10}$ Sometimes called chronic HP, these patients can have similar histopathologic and radiologic manifestations as those with idiopathic pulmonary fibrosis (IPF), ${ }^{11}$ including a usual interstitial pneumonia (UIP)-like pattern of fibrosis on lung biopsy ${ }^{5,10,12,13}$ and honeycomb cysts on high-resolution CT (HRCT) scan., ${ }^{3,14,15}$ Disease chronicity has been variably defined by duration of symptoms or evidence of pulmonary fibrosis on radiologic imaging or lung biopsy.

Despite overlap in presenting features, HP is considered to have a better prognosis than IPF. Several studies suggest that chronic HP has a longer survival time than IPF. $^{4,6}$ In contrast, when the clinical diagnosis of HP is defined by antigen exposure, patients with HP with a histopathologic UIP-like pattern have outcomes similar to unexposed UIP/IPF, ${ }^{6}$ including a median survival of 3.6 to 4.2 years, ${ }^{5,10}$ well within the range reported for IPF. $^{11}$

We sought to develop a radiologic phenotype classification associated with lung transplant-free survival and postdiagnosis pulmonary function trajectory in patients with HP. We also compare outcomes in patients with HP and IPF by radiologic phenotype. We hypothesized that after accounting for radiologic phenotype, the specific ILD diagnosis (ie, HP, IPF) provides little incremental prognostic information.

\section{Methods}

\section{Patient Selection}

Review of the University of Michigan's multidisciplinary ILD conference $^{16}$ minutes (February 1, 2009-August 31, 2014) and searching "hypersensitivity pneumonitis" International Classification of Diseases, 9th revision codes $(495.7,495.8,495.9)$ (January 1, 2004December 31, 2013) identified subjects. ILD diagnoses were verified by detailed chart review. Supporting evidence for HP diagnosis verification included a surgical biopsy reviewed by a University of Michigan pathologist as consistent with HP such that no differential diagnosis was provided (termed classic HP) (e-Table 1), or at least two of the following: BAL lymphocytosis $>20 \%$, consistent transbronchial or surgical biopsy findings (loose nonnecrotizing granulomas, giant cells, mononuclear inflammatory interstitial or peribronchiolar infiltrate named in report, or HP listed in differential diagnosis), or plausible exposure history (e-Table 1). ${ }^{1}$ e-Table 2 provides a detailed overview of supporting evidence of HP in those subjects not diagnosed via a classic HP surgical biopsy. All IPF cases had a surgical biopsy. ${ }^{11,17}$ Figure 1 shows the numbers of screened patients and reasons for exclusion. Clinical characteristics were collected from the medical record. FVC \% predicted, FEV $\%$ predicted, and diffusion capacity for carbon monoxide $\%$ predicted were measured. ${ }^{18-20}$ The date of lung transplantation or

Ann Arbor, MI; and the Division of Pulmonary and Critical Medicine (Dr Martinez), Cornell Medical College, New York, NY.

Part of this article has been presented at the Pulmonary Fibrosis Foundation Summit, November 9-11, 2017, Nashville, TN; and the American Thoracic Society International Conference, May 20-23, 2018, San Diego, CA.

FUNDING/SUPPORT: This study was funded by the Multidisciplinary Training Program in Lung Disease [Grant T32 HL00749-21], Kevin R. Flaherty [Grant K24 HL111316], and Michigan Institute for Clinical and Health Research [Grant UL1TR000433].

CORRESPONDENCE TO: Margaret L. Salisbury, MD, 1500 E Medical Center Dr, 3916 Taubman Center, Ann Arbor, MI 48109; e-mail: msalisbu@med.umich.edu

Copyright $(2018$ American College of Chest Physicians. Published by Elsevier Inc. All rights reserved.

DOI: https://doi.org/10.1016/j.chest.2018.08.1076 death without transplant was verified by medical record and the National Death Index (complete through December 31, 2016). The institutional review board at the University of Michigan approved this study (No. HUM00093978). Some results were presented as an abstract, ${ }^{21}$ and this cohort overlaps with a prior publication. ${ }^{22}$

\section{HRCT Scan Interpretation}

Interpretation methods are presented in e-Figure 1 and e-Appendix 1. Briefly, three radiologists reviewed HRCT scans. Radiologic features of honeycombing, reticular pattern, ground glass, mosaic attenuation, and air trapping (when expiratory images were available) ${ }^{23}$ were scored semi-quantitatively in each of five lobes, and lobar scores were summed and divided by five for an average (scale, 0-5). Traction bronchiectasis and centrilobular nodules were recorded as present (score $=1)$ or absent (score $=0$ ) in each lobe and summed to represent the number of lobes having the feature. A three-reader consensus average was created for semi-quantitatively scored variables. A dichotomous variable for each feature was present if the three-reader average/sum was $>0.5$. The craniocaudal distribution of disease was upper, lower, or diffuse. Axial distribution was noted as central, peripheral, subpleural, peribronchovascular, or diffuse.

\section{Statistical Methods}

Analyses were via SAS 9.4 (SAS Institute) and R 3.2.1 (The R Foundation). Baseline clinical and HRCT scan characteristics are shown as mean \pm SD, median (range), or number (\%), with comparison of characteristics in subjects with HP with subjects with IPF via $t$ test, rank-sum test, $\chi^{2}$ test, or Fisher exact test, as appropriate. Methods for measuring interobserver agreement on HRCT scan characteristics is presented in e-Appendix 1.

Survival time is calculated from the HRCT scan to composite death or lung transplant, with nonevents censored on December 31, 2016. Cox proportional hazards models assess association of predictors with survival time. To compare survival time in patients with HP with patients with IPF by radiologic phenotype and test our hypothesis that after accounting for radiologic phenotype the specific ILD diagnosis (ie, HP, IPF) provides little incremental prognostic information, we used a multivariable Cox proportional hazards model with one term capturing the radiologic phenotype-clinical diagnosis interaction. We then compared the interaction model to models with radiologic phenotype alone, clinical diagnosis alone, and adjustment variables (age, sex, FVC \% predicted) alone. The index of 


\section{1,664 Individuals from multidisciplinary ILD conference}

February 1, 2009 to August 31, 2014

415 separate individuals evaluated in UM pulmonary clinics with "HP" ICD-9 codes January 1, 2004 to December 31, 2013

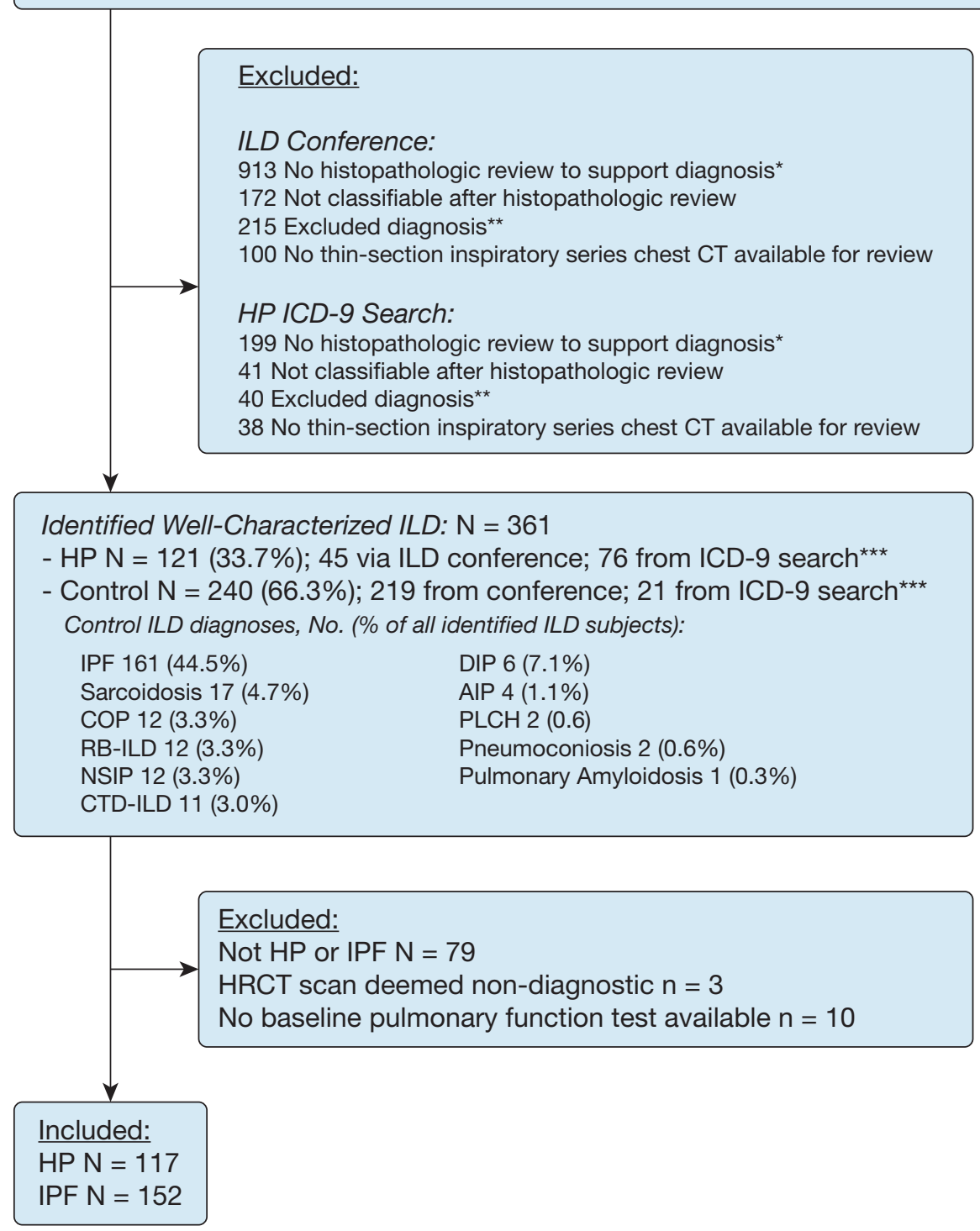

Figure 1 - Patient flow diagram detailing included subjects and exclusion criteria. ${ }^{*}$ Includes those with ILD diagnosis confirmed by HRCT scan only (ie, definite usual interstitial pneumonia HRCT scan pattern) and those unwilling or unable to undergo diagnostic biopsy. ${ }^{* *}$ Excluded diagnoses include primary cystic lung disease, pulmonary alveolar proteinosis, airway or pulmonary vascular disease without ILD, drug-induced lung disease, infection, and other non-ILD diagnoses (congestive heart failure, etc). ${ }^{* *}$ Of included patients identified from the ICD-9 search alone, $23 \mathrm{HP}$ and 7 control patients had chart documentation of multidisciplinary case review, which occurred prior to creation of a searchable database of conference minutes. $A I P=$ acute interstitial pneumonitis; $C O P=$ cryptogenic organizing pneumonia; $C T D-I L D=$ connective tissue disease-associated ILD; DIP $=$ desquamative interstitial pneumonia; $H P=$ hypersensitivity pneumonitis; $H R C T=$ high-resolution $C T$; ICD-9 = international classification of diseases, 9th revision; $I L D=$ interstitial lung disease; $I P F=$ idiopathic pulmonary fibrosis; $N S I P=$ nonspecific interstitial pneumonia; $P L C H=$ pulmonary Langerhans cell histiocytosis; RB-ILD = respiratory bronchiolitis-ILD.

concordance (c statistic) gives model discrimination. ${ }^{24} P$ values comparing $\mathrm{c}$ statistics are done via the bootstrap method. The partial likelihood ratio test (LRT) identified the most parsimonious nested model with the best fit. ${ }^{25}$ Cox model-based survival estimates with baseline hazards stratified by radiologic phenotype and clinical diagnosis, and typical patient profile assumed (age, 61.9 years; sex,
$51 \%$ women; FVC \% predicted, 65.6), are plotted to show adjusted transplant-free survival probability.

Mixed effects models estimate FVC \% predicted change over 12 months based on clinical diagnosis and radiologic phenotype, adjusted for baseline age and sex. The baseline test was dated closest 
in time to the HRCT scan. Mixed models account for loss to follow-up because of attrition using all available data measured prior to the loss to follow-up time. Hence, all 269 subjects were included in the model regardless of availability of postbaseline FVC \% predicted measurements through 12 months. FVC \% predicted measurements recorded after lung transplant were censored, and those recorded $>24$ months postbaseline were removed to mitigate survivorship bias.

\section{Results}

\section{Patient and HRCT Scan Characteristics}

Patients with HP (mean age \pm SD, $58.3 \pm 11.0$ years) were younger than those with IPF (mean age \pm SD, $64.7 \pm 8.21)$, less likely to be men ( $32 \%$ of those with HP vs $62 \%$ of those with IPF), more likely to be neversmokers (53\% of those with HP vs $39 \%$ of those with IPF), and more likely to have received corticosteroids prior to evaluation at the University of Michigan (38\% of those with HP vs $18 \%$ of those with IPF). Baseline FVC \% predicted and diffusion capacity for carbon monoxide \% predicted were similar (Table 1). Median time between the baseline pulmonary function test and HRCT scan was 7 days. e-Tables 3-6 provide detailed information about HRCT scan quality, protocols, and interrater agreement across three radiologists. As shown in Table 2, HP compared with IPF had more radiologic ground glass, mosaic attenuation, air trapping, and centrilobular nodules and less honeycombing, reticulation, and traction bronchiectasis. HP compared with IPF were more likely to have a diffuse or upper lung craniocaudal disease distribution, and diffuse axial disease distribution.

\section{HRCT Scan Phenotype and Transplant-Free Survival}

There were 137 events during 7.36 (95\% CI, 6.90-8.21) median follow-up years, including 103 deaths (HP: $\mathrm{n}=34$, IPF: $\mathrm{n}=69$ ) and 34 transplants (HP: $\mathrm{n}=9$, IPF: $\mathrm{n}=25$ ). To develop an HRCT scan phenotype associated with survival in patients with HP, the unadjusted association of clinical, physiologic, and radiologic characteristics with lung transplant-free survival in patients with HP and IPF were evaluated (Table 3). In patients with HP, presence of fibrotic features of honeycombing (hazard ratio [HR], 7.95; 95\% CI, 3.94-16.10; $P<.001)$, traction bronchiectasis (HR, 3.80; 95\% CI, 1.68-8.57; $P=.001$ ), and reticulation (HR, 9.85; 95\% CI, 4.08-23.74; $P<.001$ ) were associated with shorter survival; similar effects were observed with greater extent of these features. Three mutually exclusive radiologic phenotypes were created based on unadjusted analysis. The honeycomb phenotype is defined by presence of any honeycombing, regardless of other radiologic features.
Non-honeycomb fibrosis (termed fibrotic) is defined by absence of honeycombing but presence of traction bronchiectasis and reticulation. Both features define the fibrotic phenotype because of the greater weight of reticulation as a predictor of poor outcome and better interobserver agreement on presence of traction bronchiectasis (e-Tables 4, 6). Subjects with the nonfibrotic phenotype did not meet criteria for either of the previously described phenotype groups. eTable 7 shows relevant baseline demographics, diagnostic criteria, pulmonary function, and semiquantitative HRCT scan feature scores and postHRCT scan treatments rendered, by HRCT scan phenotype for subjects with HP. e-Table 8 shows similar data for subjects with IPF.

To determine if clinical diagnosis yielded prognostic information beyond the radiologic phenotype, a series of multivariable Cox models were assessed (Table 4). Model fit and discrimination contributed to conclusions. The base model (model 1), including age, sex, and baseline FVC \% predicted, yielded a c statistic of 0.691 (SE, 0.026). Model 2, the base model plus a clinical diagnosis variable, had a c statistic of 0.724 (SE, 0.026). Model 3, the base model plus radiologic phenotype, had a c statistic of 0.770 (SE, 0.026). Model 4 , the base model incorporating clinical diagnosis and radiologic phenotype with a clinical diagnosis by radiologic phenotype interaction, had a $c$ statistic of 0.781 (SE, 0.026). We observed a statistically significant increase in model discrimination when adding either clinical diagnosis (model 1v2, $P=.03$ ) or radiologic phenotype (model 1v3, $P<.001$ ) to the base model, with better discrimination for the radiologic phenotype than clinical diagnosis (model 2v3, $P=.01$ ). Partial LRTs similarly conclude that, separately, clinical diagnosis $(P<.001)$ and radiologic phenotype $(P<$ $.001)$ improve model fit compared with the base model alone. Adding clinical diagnosis to a model containing the base model plus radiologic phenotype did not result in a statistically significant improvement in discrimination based on the c statistic (model 3 vs 4 , $P=.13)$ but did show improved model fit by partial LRT $(P=.02)$. Model 4 (the base model plus clinical diagnosis and radiology phenotype with interaction) compared with model 2 (clinical diagnosis plus the base 
TABLE 1 ] Patient Characteristics at Baseline

\begin{tabular}{|c|c|c|c|c|}
\hline Variable & All $(N=269)$ & $H P(n=117)$ & $\operatorname{IPF}(n=152)$ & $\begin{array}{l}P \text { Value, }{ }^{\text {a }} \mathrm{HP} \\
\text { vs IPF }\end{array}$ \\
\hline Age, y & $61.9 \pm 10.0$ & $58.3 \pm 11.0$ & $64.7 \pm 8.21$ & $<.001$ \\
\hline Male sex & $133(49)$ & $38(32)$ & $95(62)$ & $<.001$ \\
\hline White race & $238(88)$ & $104(89)$ & $134(88)$ & $>.99$ \\
\hline \multicolumn{5}{|l|}{ Smoking status } \\
\hline Current & $7(3)$ & $4(3)$ & $3(2)$ & .73 \\
\hline Former & $140(52)$ & $51(44)$ & $89(59)$ & .02 \\
\hline Never & $122(45)$ & $62(53)$ & $60(39)$ & .04 \\
\hline Pack-years smoked, y ( $n=5$ missing) & $13.9 \pm 19.6$ & $11.1 \pm 17.8$ & $16.0 \pm 20.8$ & .04 \\
\hline $\begin{array}{l}\text { Reported symptom duration, median } \\
\qquad(0,25,75,100 \text { percentiles }) \text {, mo }\end{array}$ & $12(0,6,36,360)$ & $12(0,6,36,360)$ & $18(0,9,36,132)$ & $>.99$ \\
\hline $\begin{array}{l}\text { Corticosteroid treatment prior to } \\
\text { HRCT scan }\end{array}$ & $71(26)$ & $44(38)$ & $27(18)$ & $<.001$ \\
\hline \multicolumn{5}{|l|}{ Baseline physiology } \\
\hline FVC \% predicted & $65.6 \pm 16.8$ & $65.9 \pm 17.4$ & $65.4 \pm 16.3$ & .82 \\
\hline $\mathrm{FEV}_{1} \%$ predicted & $76.5 \pm 19.6$ & $73.9 \pm 20.4$ & $78.5 \pm 18.7$ & .06 \\
\hline $\mathrm{FEV}_{1} / \mathrm{FVC}$ ratio & $83.5 \pm 7.2$ & $81.9 \pm 8.5$ & $84.8 \pm 5.7$ & .002 \\
\hline $\begin{array}{l}\text { Dıco } \% \text { predicted }(n=77 \text { missing; } \\
\text { HP: } n=33, \text { IPF: } n=44)\end{array}$ & $50.6 \pm 17.4$ & $51.7 \pm 16.5$ & $49.8 \pm 18.2$ & .48 \\
\hline $\begin{array}{l}\text { Median (IQR) time between HRCT } \\
\text { scan and PFT, d }\end{array}$ & $7(0-49)$ & $7(0-40)$ & $11(0-53)$ & .83 \\
\hline \multicolumn{5}{|l|}{ Diagnostic testing available } \\
\hline Surgical lung biopsy performed & $236(88)$ & $84(72)$ & $152(100)$ & $<.001$ \\
\hline Classic HP pattern (among HP) & $\ldots$ & $62(53)$ & $\ldots$ & \\
\hline $\begin{array}{l}\text { Transbronchial lung biopsy } \\
\text { performed }\end{array}$ & $76(28)$ & $60(51)$ & $16(11)$ & $<.001$ \\
\hline BAL cell count/differential & $62(23)$ & $52(44)$ & $10(7)$ & $<.001$ \\
\hline$\%$ lymphocytes & $28.6 \pm 20.6$ & $32.8 \pm 19.7$ & $6.8 \pm 6.6$ & $<.001$ \\
\hline HP panel results & $76(28)$ & $63(54)$ & $13(9)$ & $<.001$ \\
\hline Negative $^{b}$ & $50(66)$ & $41(65)$ & $9(69)$ & \\
\hline Bird $^{\mathrm{b}}$ & $11(14)$ & $10(16)$ & $1(8)$ & \\
\hline Microbe $^{b}$ & $8(11)$ & $6(10)$ & $2(15)$ & \\
\hline Both $^{\mathrm{b}}$ & $7(9)$ & $6(10)$ & $1(8)$ & \\
\hline Reported exposures & & & & $<.001$ \\
\hline None reported & $144(53)$ & $38(32)$ & $106(70)$ & \\
\hline Asbestos & $15(6)$ & $0(0)$ & $15(10)$ & \\
\hline Beryllium & $1(0)$ & $0(0)$ & $1(1)$ & \\
\hline Bird & $32(12)$ & $32(27)$ & $1(1)$ & \\
\hline Chemical & $5(2)$ & $1(1)$ & $4(3)$ & \\
\hline Drug & $1(0)$ & $1(1)$ & $0(0)$ & \\
\hline Hot tub & $9(3)$ & $8(7)$ & $1(1)$ & \\
\hline Industrial dust & $10(4)$ & $0(0)$ & $10(7)$ & \\
\hline Microbe & $32(12)$ & $24(20)$ & $8(5)$ & \\
\hline Multiple & $11(4)$ & $10(8)$ & $1(1)$ & \\
\hline Wood dust & $8(3)$ & $3(3)$ & $5(3)$ & \\
\hline
\end{tabular}

Values are mean $\pm S D$, No. (\%), or as otherwise indicated. DLCo = diffusion capacity for carbon monoxide; HP = hypersensitivity pneumonitis; HRCT = high-resolution CT; IPF = idiopathic pulmonary fibrosis; IQR = interquartile range; PFT = pulmonary function test.

a $P$ values compare HP with IPF using a $t$ test or $\chi^{2}$ test, as appropriate. In cases where a median (IQR) or median (range) is presented, the nonparametric (rank-sum) test was used.

bThe denominator for the proportion with the named result is the total of those with the test performed. 
TABLE 2 ] Summary of Consensus HRCT Scan Features for All Subjects and for Subjects With HP and IPF

\begin{tabular}{|c|c|c|c|c|}
\hline \multirow[b]{2}{*}{ Variable } & \multicolumn{4}{|c|}{ Group } \\
\hline & All Subjects $(N=269)$ & $\mathrm{HP}(\mathrm{n}=117)$ & $\operatorname{IPF}(n=152)$ & $P$ Value, HP vs IPF \\
\hline \multicolumn{5}{|l|}{ Semi-quantitative scores ${ }^{a}$} \\
\hline Honeycombing & $0.23 \pm 0.49$ & $0.16 \pm 0.45$ & $0.29 \pm 0.52$ & .03 \\
\hline Reticulation & $0.93 \pm 0.51$ & $0.63 \pm 0.54$ & $1.17 \pm 0.33$ & $<.001$ \\
\hline Ground glass & $1.59 \pm 1.01$ & $1.99 \pm 1.21$ & $1.28 \pm 0.69$ & $<.001$ \\
\hline Mosaic attenuation & $0.56 \pm 0.87$ & $1.04 \pm 1.06$ & $0.20 \pm 0.43$ & $<.001$ \\
\hline Air trapping $(n=229)$ & $0.89 \pm 0.88$ & $1.40 \pm 0.92$ & $0.48 \pm 0.58$ & $<.001$ \\
\hline Centrilobular nodules & $0.40 \pm 0.89$ & $0.74 \pm 1.16$ & $0.13 \pm 0.45$ & $<.001$ \\
\hline Traction bronchiectasis & $3.16 \pm 1.73$ & $1.93 \pm 1.80$ & $4.11 \pm 0.88$ & $<.001$ \\
\hline \multicolumn{5}{|l|}{ Dichotomous scores $^{\mathrm{b}}$} \\
\hline Honeycombing & $41(15)$ & $12(10)$ & $29(19)$ & .07 \\
\hline Reticulation & $212(79)$ & $61(52)$ & 151 (99) & $<.001$ \\
\hline Ground glass & $242(90)$ & $109(92)$ & $133(88)$ & .18 \\
\hline Mosaic attenuation & $89(33)$ & $66(56)$ & $23(15)$ & $<.001$ \\
\hline Air trapping $(n=229)$ & $124(54)$ & $80(78)$ & $44(35)$ & $<.001$ \\
\hline Mosaic attenuation or air trapping & $144(53)$ & $91(77)$ & $53(35)$ & $<.001$ \\
\hline Centrilobular nodules & $61(23)$ & $46(39)$ & $15(10)$ & $<.001$ \\
\hline Traction bronchiectasis & $229(85)$ & $77(65)$ & $152(100)$ & $<.001$ \\
\hline \multicolumn{5}{|c|}{ Craniocaudal distribution, average Probability } \\
\hline Upper lung & $0.09 \pm 0.22$ & $0.18 \pm 0.30$ & $0.02 \pm 0.08$ & $<.001$ \\
\hline Lower lung & $0.59 \pm 0.42$ & $0.32 \pm 0.39$ & $0.81 \pm 0.31$ & $<.001$ \\
\hline Diffuse & $0.31 \pm 0.36$ & $0.50 \pm 0.38$ & $0.17 \pm 0.28$ & $<.001$ \\
\hline \multicolumn{5}{|l|}{ Axial distribution, average probability } \\
\hline Central lung & $0.01 \pm 0.09$ & $0.02 \pm 0.11$ & $0.01 \pm 0.09$ & $<.001$ \\
\hline Peripheral lung & $0.55 \pm 0.41$ & $0.29 \pm 0.37$ & $0.76 \pm 0.30$ & $<.001$ \\
\hline Subpleural & $0.02 \pm 0.09$ & $0.01 \pm 0.06$ & $0.04 \pm 0.10$ & .02 \\
\hline Peribronchovascular & $0.00 \pm 0.03$ & $0.00 \pm 0.03$ & $0.00 \pm 0.03$ & .86 \\
\hline Diffuse & $0.38 \pm 0.39$ & $0.62 \pm 0.39$ & $0.19 \pm 0.28$ & $<.001$ \\
\hline
\end{tabular}

Values are mean \pm SD, No. (\%), or as otherwise indicated. See Table 1 legend for expansion of abbreviations.

aSemi-quantitative scores: For honeycombing, reticulation, ground glass, mosaic attenuation, and air trapping, the average score was determined by summing five lobe scores and dividing by five for each subject and each radiologist/reader. For centrilobular nodules and traction bronchiectasis, the sum was determined by adding the score for five lobes. The three radiologist's scores were then averaged. Average and sum scores are scaled from 0 to 5 , corresponding to the proportion of total lung having the feature (for honeycombing, reticulation, ground glass, mosaic attenuation, air trapping) or number of lobes having the feature (centrilobular nodules, traction bronchiectasis).

bDichotomous scores: A feature was designated as absent when the three-reader average score (for honeycombing, reticulation, ground glass, mosaic attenuation, air trapping) or sum score (centrilobular nodules, traction bronchiectasis) for that features was $<0.5$, otherwise the feature was considered present.

model) had significantly better fit and discrimination (c statistic, $P<.001$; partial LRT, $P<.001$ ).

Using model 4 , transplant-free survival time was compared by diagnosis and phenotype group (Fig 2, Table 5). Within subjects with HP, radiologic phenotype group had distinct survival times: patients with nonfibrotic HP survive longer than those with fibrotic HP (HR, 0.22; 95\% CI, 0.10-0.51; P<.001) and honeycomb HP (HR, 0.06; 95\% CI, 0.02-0.15;
$P<.001$ ), and subjects with fibrotic HP survive longer than those with honeycomb HP (HR, 0.26; 95\% CI, $0.12-0.54 ; P<.001)$. Similarly, subjects with fibrotic IPF survive longer than those with honeycomb IPF (HR, 0.59; 95\% CI, 0.37-0.95; $P=.03$ ). Within the fibrotic phenotype, subjects with HP had significantly longer survival than those with IPF (HR, 0.46; 95\% CI, $0.27-0.77 ; P=.003)$. In a separate model including only subjects with the fibrotic phenotype, an IPF 
TABLE 3 ] Unadjusted Cox Analysis: Clinical, Physiologic, and Radiologic Variables

\begin{tabular}{|c|c|c|c|c|}
\hline \multirow[b]{2}{*}{ Predictor } & \multicolumn{2}{|c|}{$\mathrm{HP}(\mathrm{n}=117)$} & \multicolumn{2}{|c|}{$\operatorname{IPF}(n=152)$} \\
\hline & HR $(95 \%$ CI) & $P$ Value & HR $(95 \%$ CI) & $P$ Value \\
\hline Age, per additional 1 year & $1.05(1.02-1.08)$ & .002 & $1.01(0.98-1.04)$ & .46 \\
\hline Sex (male vs others) & $0.85(0.45-1.61)$ & .62 & $1.06(0.70-1.62)$ & .77 \\
\hline Race (white vs others) & $1.06(0.38-2.97)$ & .91 & $0.98(0.51-1.90)$ & .96 \\
\hline Never-smoker status (vs ever) & $0.73(0.40-1.35)$ & .32 & $0.63(0.41-0.97)$ & .04 \\
\hline Pack-years smoked, per additional 1 year & $1.01(0.99-1.02)$ & .31 & $0.999(0.99-1.01)$ & .83 \\
\hline Reported symptom duration, per additional 1 month & $1.00(0.999-1.01)$ & .15 & $1.00(0.99-1.01)$ & .53 \\
\hline Corticosteroid prior consult & $1.61(0.88-2.94)$ & .12 & $1.37(0.83-2.27)$ & .22 \\
\hline \multicolumn{5}{|l|}{ Baseline physiology, per additional $1 \%$} \\
\hline FVC \% predicted & $0.99(0.97-1.004)$ & .12 & $0.96(0.95-0.98)$ & $<.001$ \\
\hline $\mathrm{FEV}_{1} \%$ predicted & $0.99(0.98-1.01)$ & .37 & $0.97(0.96-0.98)$ & $<.001$ \\
\hline $\mathrm{FEV}_{1} / \mathrm{FVC}$ ratio & $1.02(0.98-1.07)$ & .28 & $1.04(1.004-1.08)$ & .03 \\
\hline DLco \% predicted ${ }^{a}$ & $0.99(0.96-1.01)$ & .21 & $0.95(0.94-0.97)$ & $<.001$ \\
\hline Honeycombing dichotomous & $7.95(3.94-16.10)$ & $<.001$ & $1.86(1.17-2.95)$ & $<.001$ \\
\hline Honeycombing SQ ${ }^{\mathrm{b}}$ & $4.87(2.96-8.01)$ & $<.001$ & $1.63(1.21-2.20)$ & .001 \\
\hline Reticulation dichotomous & $9.85(4.08-23.74)$ & $<.001$ & $n / a^{c}$ & $\mathrm{n} / \mathrm{a}^{\mathrm{c}}$ \\
\hline Reticulation $\mathrm{SQ}^{\mathrm{b}}$ & $6.91(3.84-12.43)$ & $<.001$ & $3.61(1.93-6.74)$ & $<.001$ \\
\hline Ground glass dichotomous & $3.69(0.51-26.89)$ & .20 & $1.10(0.60-2.02)$ & .76 \\
\hline Ground glass $\mathrm{SQ}^{\mathrm{b}}$ & $0.81(0.64-1.05)$ & .11 & $1.18(0.89-1.57)$ & .24 \\
\hline Mosaic attenuation dichotomous & $0.67(0.37-1.21)$ & .18 & $1.33(0.77-2.27)$ & .31 \\
\hline Mosaic attenuation $\mathrm{SQ}^{\mathrm{b}}$ & $1.04(0.78-1.40)$ & .77 & $1.08(0.68-1.72)$ & .74 \\
\hline Air trapping dichotomous ${ }^{a}$ & $0.66(0.32-1.36)$ & .26 & $1.05(0.67-1.65)$ & .83 \\
\hline Air trapping $\mathrm{SQ}^{\mathrm{a}, \mathrm{b}}$ & $0.87(0.60-1.25)$ & .44 & $1.14(0.79-1.65)$ & .47 \\
\hline MA-AT dichotomous & $0.62(0.32-1.21)$ & .16 & $1.13(0.75-1.72)$ & .55 \\
\hline Centrilobular nodules dichotomous & $0.33(0.16-0.70)$ & .003 & $1.41(0.77-2.59)$ & .27 \\
\hline Centrilobular nodules $\mathrm{SQ}^{\mathrm{b}}$ & $0.54(0.34-0.84)$ & .006 & $1.33(0.91-1.95)$ & .14 \\
\hline Traction bronchiectasis dichotomous & $3.80(1.68-8.57)$ & .001 & $n / a^{c}$ & $\mathrm{n} / \mathrm{a}^{\mathrm{c}}$ \\
\hline Traction bronchiectasis $\mathrm{SQ}^{\mathrm{b}}$ & $1.65(1.37-2.00)$ & $<.001$ & $1.996(1.50-2.67)$ & $<.001$ \\
\hline \multicolumn{5}{|l|}{ Craniocaudal distribution } \\
\hline Upper & $0.78(0.23-2.61)$ & .67 & $0.31(0.02-4.43)$ & .39 \\
\hline Lower & $1.25(0.54-2.90)$ & .60 & $0.58(0.28-1.18)$ & .13 \\
\hline Diffuse (REF) & $\ldots$ & $\ldots$ & $\ldots$ & $\ldots$ \\
\hline \multicolumn{5}{|l|}{ Axial distribution } \\
\hline Central or peribronchovascular & $n / a^{c}$ & $\mathrm{n} / \mathrm{a}^{\mathrm{c}}$ & $0.74(0.09-6.34)$ & .78 \\
\hline Peripheral/subpleural & $2.46(1.33-4.58)$ & .004 & $0.87(0.35-2.14)$ & .76 \\
\hline Diffuse (REF) & $\ldots$ & $\ldots$ & $\ldots$ & $\ldots$ \\
\hline
\end{tabular}

$\mathrm{HR}=$ hazard ratio; MA-AT = combined variable denoting presence of mosaic attenuation or air trapping; $\mathrm{n} / \mathrm{a}=$ not applicable; REF = reference; $\mathrm{SQ}=$ semi-quantitative. See Table 1 legend for expansion of other abbreviations.

${ }^{a}$ Missing data: 77 subjects did not have a baseline diffusion capacity for carbon monoxide \% predicted measurement, including 33 patients with HP and 44 patients with IPF. There were 229 subjects who had an expiratory image series for evaluation of air trapping, including 102 patients with HP and 127 patients with IPF.

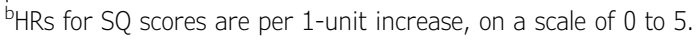

'HR was not calculable because all subjects with IPF have traction bronchiectasis, 99\% have reticulation, and there are no events in subjects with HP with central or peribronchovascular disease distribution.

diagnosis was associated with shortened survival (HR, 1.45; 95\% CI, 0.88-2.97; $P=.13$ ) after adjusting for fibrosis extent using the traction bronchiectasis score, age, sex, and FVC \% predicted (e-Table 9); however, the effect did not reach statistical significance. Within the honeycomb phenotype, subjects with HP had 
TABLE 4 ] Nested Multivariable Cox Analysis

\begin{tabular}{|c|c|c|c|c|}
\hline Predictor & $\begin{array}{c}\text { Model } 1 \\
\text { (c statistic = 0.691; } \\
\text { SE, 0.026) }\end{array}$ & $\begin{array}{c}\text { Model } 2 \\
\text { (c statistic }=0.724 ; \\
\mathrm{SE}=0.026)\end{array}$ & $\begin{array}{c}\text { Model } 3 \\
\text { (c statistic = 0.770; } \\
\mathrm{SE}=0.026 \text { ) }\end{array}$ & $\begin{array}{c}\text { Model } 4 \\
\text { (c statistic }=0.781 ; \\
\mathrm{SE}=0.026 \text { ) }\end{array}$ \\
\hline Female sex & $\begin{array}{c}0.77 \\
(0.54-1.07 ; .11)\end{array}$ & $\begin{array}{c}1.07 \\
(0.75-1.54 ; .70)\end{array}$ & $\begin{array}{c}0.98 \\
(0.69-1.38 ; .89)\end{array}$ & $\begin{array}{c}1.15 \\
(0.78-1.65 ; .46)\end{array}$ \\
\hline Age, per 1-year increase & $\begin{array}{c}1.05 \\
(1.03-1.07 ;<.001)\end{array}$ & $\begin{array}{c}1.03 \\
(1.02-1.05 ;<.001)\end{array}$ & $\begin{array}{c}1.03 \\
(1.01-1.05 ; .003)\end{array}$ & $\begin{array}{c}1.03 \\
(1.01-1.05 ; .008)\end{array}$ \\
\hline $\begin{array}{l}\text { FVC } \% \text { predicted, } \\
\text { per } 1 \% \text { increase }\end{array}$ & $\begin{array}{c}0.97 \\
(0.96-0.98 ;<.001)\end{array}$ & $\begin{array}{c}0.97 \\
(0.96-0.98 ;<.001)\end{array}$ & $\begin{array}{c}0.97 \\
(0.96-0.98 ;<.001)\end{array}$ & $\begin{array}{c}0.97 \\
(0.96-0.98 ;<.001)\end{array}$ \\
\hline IPF diagnosis (vs HP) & $\ldots$ & $\begin{array}{c}3.10 \\
(2.00-4.79 ;<.001)\end{array}$ & $\cdots$ & $\cdots$ \\
\hline Radiologic phenotype & $\ldots$ & $\ldots$ & & $\ldots$ \\
\hline Nonfibrotic $(n=61)$ & & & $\begin{array}{c}0.15 \\
(0.07-0.31 ;<.001)\end{array}$ & \\
\hline Fibrotic $(n=167)$ & & & REF & \\
\hline Honeycombing $(n=41)$ & & & $\begin{array}{c}2.12 \\
(1.42-3.17 ;<.001)\end{array}$ & \\
\hline Clinical-radiologic group & $\ldots$ & $\ldots$ & $\ldots$ & \\
\hline IPF-nonfibrotic $(\mathrm{n}=1)$ & & & & $\mathrm{n} / \mathrm{a}^{\mathrm{a}}$ \\
\hline HP-nonfibrotic $(n=60)$ & & & & REF \\
\hline IPF-fibrotic $(n=122)$ & & & & $\begin{array}{c}9.64 \\
(4.38-21.24 ;<.001)\end{array}$ \\
\hline HP-fibrotic $(n=45)$ & & & & $\begin{array}{c}4.01 \\
(1.95-9.98 ;<.001)\end{array}$ \\
\hline IPF-honeycomb $(n=29)$ & & & & $\begin{array}{c}16.27 \\
(6.86-38.59 ;<.001)\end{array}$ \\
\hline HP-honeycomb $(n=12)$ & & & & $\begin{array}{c}17.18 \\
(6.82-43.27 ;<.001)\end{array}$ \\
\hline
\end{tabular}

Values are hazard ratio (95\% CI; $P$ value). Pairwise $P$ value comparisons of c statistic for model 1 vs $2=.03$; model 1 vs $3<.001$; model 1 vs $4<.001$; model 2 vs $3=.01$; model 2 vs $4<.001$; and model 3 vs $4=.13$. Likelihood ratio test $P$ value model 1 vs $2<.001$; model 1 vs $3<.001$; model 1 vs $4<$ .001 ; model 2 vs $4<.001$; and model 3 vs $4=.02$. Please note that models 2 and 3 are not nested within each other. See Table 1 and 3 legends for expansion of other abbreviations.

aToo few subjects/events for reliable comparisons.

similar survival time (HR, 1.06; 95\% CI, 0.50-2.22; $P=.89$ ) compared with subjects with IPF (Table 5). In a separate model evaluating only subjects with the honeycomb phenotype, this conclusion was robust after adjusting for traction bronchiectasis score, age, sex, and baseline FVC \% predicted (e-Table 10).

\section{HRCT Scan Phenotype and Pulmonary Function Trajectory}

In total, 269 subjects had 1,159 FVC \% predicted measurements, with a mean 4.3 measurements per subject (range, 1-15). A total of 220 subjects had at least two measurements. In unadjusted analysis, subjects who were nonfibrotic had a higher baseline FVC \% predicted than other groups (e-Tables 7,8 ). In adjusted FVC $\%$ predicted trajectory analyses for 12 months postbaseline (Fig 3), subjects with nonfibrotic HP experienced a $1.92 \%$ gain in FVC \% predicted (95\% CI, $0.49-3.35 ; P=.009)$. Subjects with fibrotic HP $(-1.65$; $95 \% \mathrm{CI},-3.19$ to $-0.10 ; P=.04)$ and honeycomb HP $(-6.80 \%$; $95 \%$ CI, -10.57 to $-3.04 ; P<.001)$ had declining FVC \% predicted. Aside from one subject with radiologically nonfibrotic IPF, subjects with IPF experienced pulmonary function decline: fibrotic by $-3.86 \%(95 \% \mathrm{CI},-4.83$ to $-2.89 ; P<.001)$ and honeycomb by $-4.13 \%$ ( $95 \% \mathrm{CI},-6.39$ to -1.87 ; $P<.001)$.

\section{Discussion}

In a cohort of subjects with HP and IPF, we identified three radiologically defined phenotypes among subjects with HP. Phenotype groups have distinct postdiagnosis survival time and pulmonary function trajectory. Radiologic honeycombing is associated with the shortest median survival (2.8 years) in HP, 


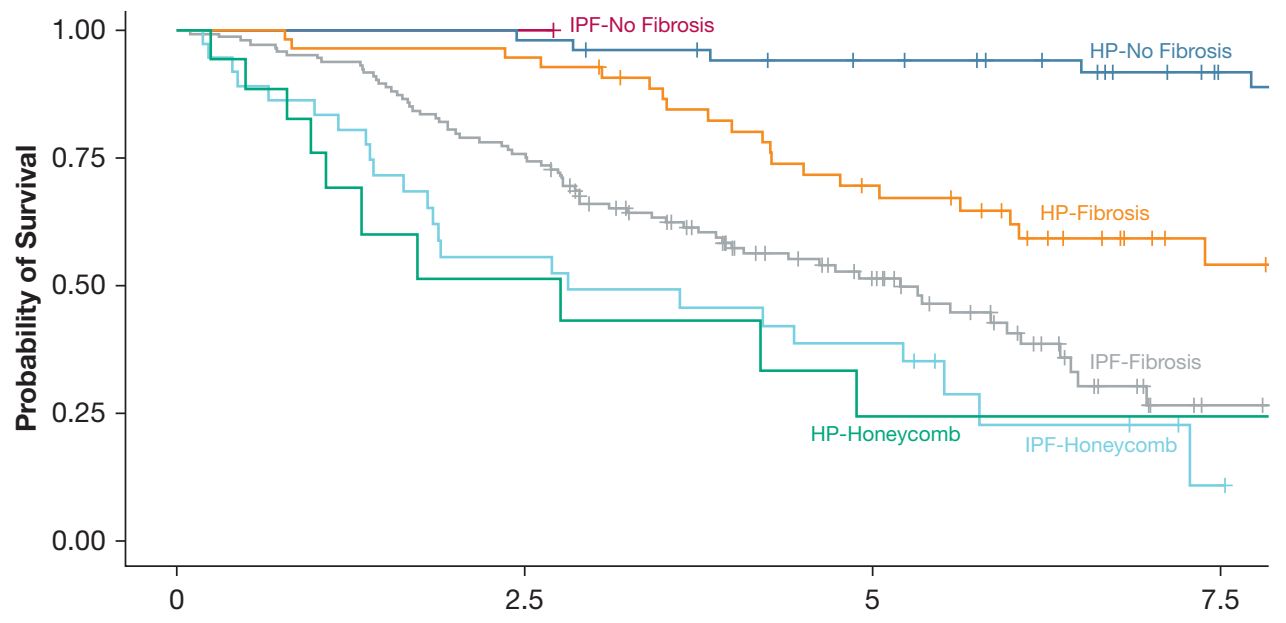

No. at risk at each time point (time in years)

\begin{tabular}{rcccc|}
\cline { 2 - 4 } IPF-No Fibrosis - & 1 & 1 & 0 & 0 \\
HP-No Fibrosis - & 60 & 59 & 53 & 41 \\
IPF-Fibrosis - & 122 & 89 & 40 & 5 \\
HP-Fibrosis - & 45 & 42 & 27 & 11 \\
IPF-Honeycomb - & 29 & 14 & 9 & 1 \\
HP-Honeycomb - & 12 & 5 & 2 & 2 \\
\hline
\end{tabular}

Figure 2 - Cox model-based survival estimates by clinical diagnosis and radiologic phenotype group. The plot shows that patients with HP without radiologic fibrosis have significantly longer lung transplant-free survival (median, $>14.73 y$ ) than patients with HP (median, $7.95 y)$ and IPF (median, $5.20 y$ ) with non-honeycomb fibrosis, and patients with HP (median, $2.76 y$ ) and IPF (median, 2.81 y) with radiologic honeycombing. The group of subjects with IPF without fibrosis was small $(n=1)$, and median survival could not be estimated. Non-honeycomb fibrosis is defined by absence of honeycombing but presence of both traction bronchiectasis and reticulation. The honeycomb groups are defined by presence of honeycombing on highresolution CT scan. The plot is adjusted for a typical age $(61.9 \mathrm{y})$, sex (51\% women), and FVC \% predicted (65.6\%) profile. See Figure 1 legend for expansion of abbreviations.

similar to honeycomb IPF (2.8 years). HP subjects with non-honeycomb fibrosis had intermediate survival (7.95 years), significantly better than those with fibrotic IPF (5.2 years). HP subjects without radiologic fibrosis survived longest ( $>14.7$ years) and experienced significant 12-month improvement in FVC \% predicted. Knowledge of clinical diagnosis in addition to radiologic phenotype resulted in statistically significant improvement in fit of a Cox proportional hazards model, likely driven by longer survival among subjects with fibrotic HP vs subjects with fibrotic IPF. Given the uniformly poor outcome among subjects with radiologic honeycombing, pursuit of invasive diagnostic tests directed at differentiating IPF from HP may be of limited value. Conversely, subjects with non-honeycomb fibrosis HP had significantly longer survival than subjects with IPF despite both demonstrating radiologic non-honeycomb fibrosis, underlining the importance of assigning the correct clinical diagnosis in patients with non-honeycomb fibrosis on HRCT scan. Despite longer survival among patients with HP, patients with fibrotic
HP and IPF had a similar 12-month FVC \% predicted decline.

Numerous analyses highlight the prognostic value of identification and/or quantification of radiologic fibrosis among patients with HP..$^{3,4,26,27}$ Mooney et al ${ }^{4}$ found that greater extent of radiologic fibrosis (measured semi-quantitatively) is associated with shortened transplant-free survival among patients with HP. Here, a clinical diagnosis of IPF and radiologic fibrosis score were independent predictors of increased hazard of death/transplant. Our results are in line with those of Mooney et al, but suggest there is value in separating subjects with radiologic honeycombing from those with non-honeycomb fibrosis given a particularly poor prognosis in the face of radiologic honeycombing, a reliable marker of histopathologic UIP. ${ }^{11}$ Perez-Padilla et al $^{6}$ compared survival among bird-exposed subjects with HP (ie, those with histopathologic findings of interstitial/ peribronchiolar mononuclear inflammation and giant cells/loose nonnecrotizing granuloma), bird-exposed subjects with biopsy-proved UIP, and unexposed 


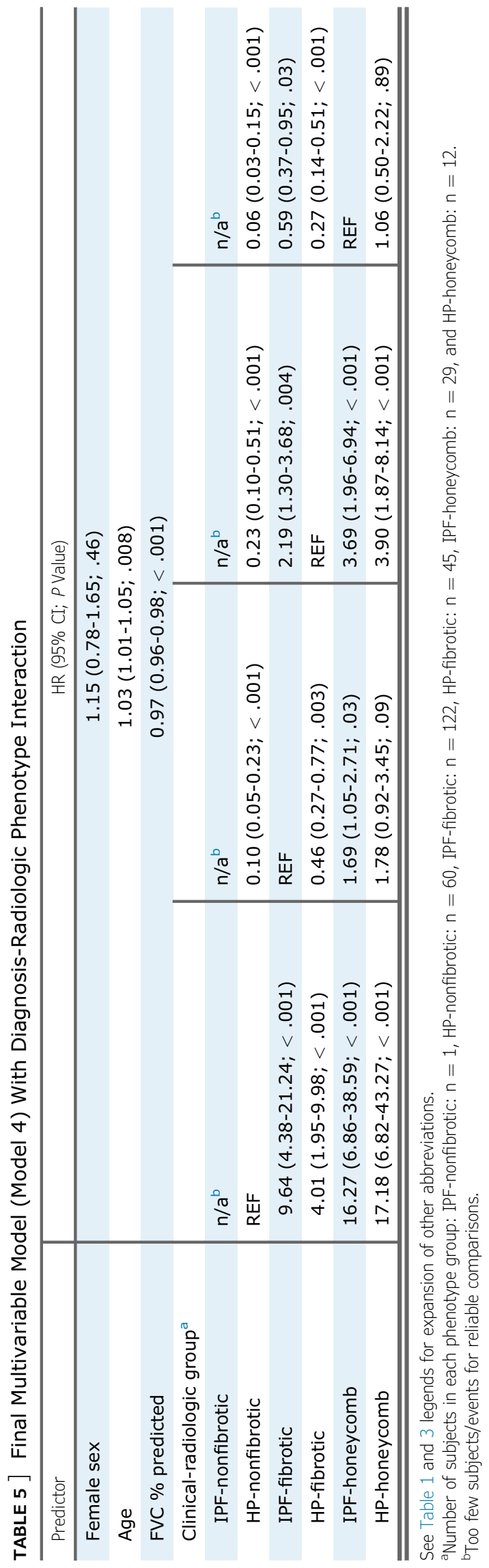

subjects with UIP/IPF. Survival among bird-exposed and unexposed UIP was nearly identical, with subjects with biopsy-proved HP surviving significantly longer than subjects with UIP. ${ }^{6}$ Survival analyses comparing a variety of ILD categories with UIP/IPF similarly demonstrate that patients displaying histopathologic or radiologic UIP have similar survival as UIP/IPF, even in the presence of autoantibodies (as in interstitial pneumonia with autoimmune features), ${ }^{28}$ a diagnosis of connective tissue disease such as rheumatoid arthritis, ${ }^{29}$ or in the presence of an environmental exposure associated with HP. ${ }^{6}$ Although we display HP honeycomb as a subcategory of HP, one must wonder if it would be better categorized in the same class as UIP/ IPF.

Additional study is needed to clarify the prognostic significance of a reported environmental exposure, BAL lymphocytosis, and/or minor biopsy findings such as granulomas, giant cells, or airway disease among patients with radiologic honeycombing or histopathologic UIP. Also unclear is whether individuals with radiologic honeycombing or histopathologic UIP and clinical/laboratory findings suggesting HP display different disease biology or treatment response compared with UIP/IPF.

Importantly, radiologic honeycombing was defined in this study as a three-reader averaged semi-quantitative honeycombing score $>0.5$. Therefore, some subjects in the non-honeycomb groups may have had minimal honeycombing (eg, involving $\leq 5 \%$ of a lobe) as noted by one or more readers. Indeed, as showed in e-Table 7 among the subjects with fibrotic HP, the average semiquantitative score for honeycombing was 0.04 , and in e-Table 8 among subjects with fibrotic IPF, the average semi-quantitative score for honeycombing was 0.09 .

Chung et $\mathrm{al}^{26}$ evaluated the prognostic value of various radiologic features and found that presence of radiologic fibrosis (including honeycombing) is associated with significantly increased hazard of death among HP. Another relevant finding in that study is an association of mosaic attenuation or air trapping with reduced hazard of death. In our study, in the HP cohort, dichotomized mosaic attenuation or air trapping was associated with a nonsignificantly reduced hazard of death/transplant. Lack of power in our study or different accounting for transplant events in survival models could explain discrepant results. $^{26}$ 


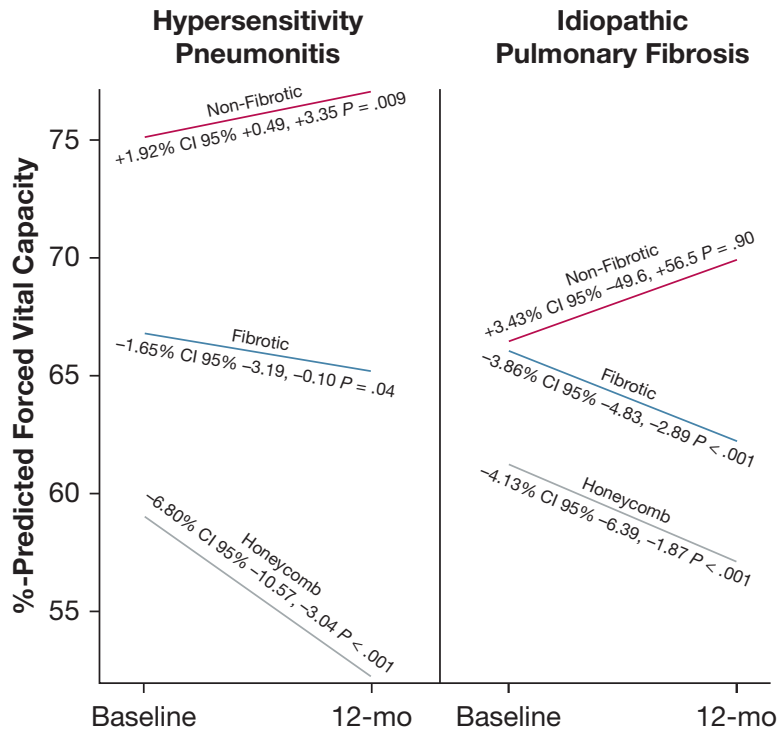

Figure 3 - FVC trajectory by clinical diagnosis and radiologic phenotype group. The plot shows that patients with hypersensitivity pneumonitis without radiologic fibrosis have significant increase in FVC \% predicted, whereas all other groups experience a significant decrease in FVC $\%$ predicted. The group of subjects with idiopathic pulmonary fibrosis without fibrosis was small $(n=1)$ making comparisons difficult. The plot is adjusted for a typical age (61.9 y) and sex (51\% women) profile. The baseline FVC \% predicted location is therefore slightly different than the unadjusted values by diagnosis and phenotype group cited in the text.

Clinical classification of HP has historically been based on disease duration (eg, acute, subacute, chronic), ${ }^{1,30-32}$ with little evidence that these distinctions provide clinically useful information in terms of selection of drug therapies, monitoring response to treatment, or prognostication. We retrospectively collected information on reported duration of symptoms prior to consultation at our center and found that symptom duration was not associated with unadjusted survival time (HR, 1.0 per additional month of symptoms; 95\% CI, 0.9991.01; $P=.15$ among HP). Further, e-Table 7 indicates that symptom duration is similar across radiologic phenotypes among subjects with HP: nonfibrotic median was 12 (range, 0-120) months, fibrotic HP was 12 (range, 2-120) months, and honeycomb HP was 13 (range, 1-360) months. Lima et $\mathrm{al}^{33}$ documented similar findings (HR, 1.01 per additional month of symptoms; 95\% CI, 0.99-1.02; $P=.57$ ). Vourlekis et $\mathrm{al}^{8}$ found similar duration of symptoms in subjects who were alive (mean $\pm \mathrm{SD}$, $2.4 \pm 2.7$ years) and dead (mean $\pm \mathrm{SD}, 2.5 \pm 2.6$ years). Ohtani et $\mathrm{al}^{5}$ documented similar symptom duration in subjects with HP with cellular nonspecific interstitial pneumonitis-like or bronchiolitis obliterans with organizing pneumonia-like histopathologic patterns (mean \pm SD, $19.3 \pm 7.5$ months) compared with those with UIP-like patterns (mean \pm SD, $24.2 \pm 5.3$ months), despite significantly shorter median survival in UIP. Taken with prior reports, our results raise concern that classification of HP by symptom duration for research or clinical purposes may be misdirected. A preponderance of evidence indicates that objective assessment of fibrosis status (using histopathologic or radiologic measure) is useful in considering prognosis, and possibly treatment decisions, in patients with HP. ${ }^{1-5,7-9,26,27,34}$

Our study has weaknesses. First, requiring a lung biopsy for IPF diagnosis may select subjects with atypical clinical or radiologic features compared with IPF all-comers. However, we deemed this bias more suitable than the alternative of including uncertain IPF diagnoses (ie, unbiopsied IPF), given the research question. Of note, IPF with honeycombing has less extensive ground glass, mosaic attenuation, air trapping, and centrilobular nodules than fibrotic IPF (e-Table 8), mitigating concern about atypical CT scan features. Second, subjects with HP and IPF referred to our quaternary center may differ systematically compared with the broader population (eg, referral for diagnostic/treatment dilemma). Third, these results should be verified in an independent cohort prior to broad clinical application. Fourth, we do not specifically account for postdiagnosis treatment effects on survival or pulmonary function change. Patients with fibrotic or honeycomb HP were more likely to receive immunosuppressant therapy than patients with IPF, and patients with IPF were more likely to receive antifibrotic or no therapy compared with HP. In the nonfibrotic HP group, only $13 \%$ received no treatment after diagnosis.

In conclusion, we identify three radiologically defined subgroups of patients with HP with unique prognoses. Those not meeting our radiologic criteria for pulmonary fibrosis (based on absence of radiologic honeycombing and traction bronchiectasis/ reticulation) experienced improving pulmonary function in 12 months after diagnosis and had significantly longer lung transplant-free survival than other subjects with HP. Those with fibrotic HP but no radiologic honeycombing had longer survival than patients with fibrotic IPF, but experienced a 12 month decline in pulmonary function. Those with radiologic honeycombing had poor median survival and declining 
FVC \% predicted regardless of clinical diagnosis. These results highlight the importance of making a correct diagnosis of HP (vs IPF) in patients with non-honeycomb fibrosis and the limited utility in differentiating HP from IPF among patients with radiologic honeycombing.

\section{Acknowledgments}

Author contributions: M. L. S. takes full responsibility for the content of this manuscript, including data and analysis. $\mathrm{M}$. L. S., K. R. F., F. J. M., and E. A. K. conceived and designed the study. M. L. S. and T. G. analyzed the data with supervision from S. M. K. R. F., F. J. M., E. A. K., B. H. G., A. C., M. S., J. L. M., A. L., K. E. K., E. A. B., J. S. S., E. S. W., and C. H. contributed data. M. L. S. prepared the manuscript. All authors critically revised the manuscript for intellectual content, approved the final draft, and agree to accountability for all aspects of the work.

Financial/nonfinancial disclosures: The authors have reported to CHEST the following: M. L. S. reports grants from the National Institutes of Health (NIH), during the conduct of the study. S. M. reports grants from the NIH, during the conduct of the study. F. J. M. reports grants from the NIH; nonfinancial support from Bayer, Centocor, Gilead, and Promedior; personal fees from Ikaria, Genentech, Nycomed/Takeda, Pfizer, Vertex, American Thoracic Society, Inova Health System, MedScape, Spectrum Health System, University of Texas Southwestern, Stromedix/Biogen, Axon Communications, Johnson \& Johnson, Genzyme, National Association for Continuing Education, Boehringer Ingelheim, and Veracyte, during the conduct of the study; and personal fees from Forest, Janssens, GSK, Nycomed/ Takeda, Actelion, Amgen, Astra Zeneca, CSA Medical, Ikaria/Bellerophon, Genentech, Merck, Pearl, Pfizer, Roche, Sudler \& Hennessey, American College of Chest Physicians, CME Incite, Center for Healthcare Education, Inova Health System, MedScape, Miller Medical, National Association for Continuing Education, Paradigm, Peer Voice, Projects in Knowledge, St. John's Hospital, St. Mary's Hospital, University of Illinois Chicago, UpToDate, Wayne State University, Boehringer Ingelheim, Ikaria, Bayer, Grey Healthcare, Merion, Informa, and Annenberg, outside the submitted work. K. R. F. reports grants from the NIH, during the conduct of the study; personal fees from Boehringer Ingelheim, Fibrogen, Genentech, Gilead, Ikaria, Intermune, ImmuneWorks, MedImmune, Novartis, Takeda, Vertex, Veracyte, Roche, and Pulmonary Fibrosis Foundation; and grants from ImmuneWorks, Intermune, and Bristol-Myers Squibb, outside the submitted work. None declared (T. G., B. H. G., A. C., M. S., E. A. K., J. L. M., A. L., K. E. K., E. A. B., J. S. S., E. S. W., C. H.).

Role of sponsors: The funding agencies provided funds to support author salaries and data analysis but had no role in study conceptualization or manuscript preparation.

Additional information: The e-Appendix, e-Figure, and e-Tables can be found in the Supplemental Materials section of the online article.

\section{References}

1. Salisbury ML, Myers JL, Belloli EA, Kazerooni EA, Martinez FJ, Flaherty KR. Diagnosis and treatment of fibrotic hypersensitivity pneumonia. Where we stand and where we need to go. Am J Respir Crit Care Med. 2017;196(6):690699.

2. Churg A, Sin DD, Everett D, Brown K, Cool C. Pathologic patterns and survival in chronic hypersensitivity pneumonitis. Am J Surg Pathol. 2009;33(12):1765-1770.

3. Hanak V, Golbin JM, Hartman TE, Ryu JH. High-resolution CT findings of parenchymal fibrosis correlate with prognosis in hypersensitivity pneumonitis. Chest. 2008;134(1):133-138.

4. Mooney JJ, Elicker BM, Urbania TH, et al. Radiographic fibrosis score predicts survival in hypersensitivity pneumonitis. Chest. 2013;144(2):586-592.

5. Ohtani Y, Saiki S, Kitaichi M, et al. Chronic bird fancier's lung: histopathological and clinical correlation. An application of the 2002 ATS/ERS consensus classification of the idiopathic interstitial pneumonias. Thorax. 2005;60(8):665-671.

6. Perez-Padilla R, Salas J, Chapela R, et al. Mortality in Mexican patients with chronic pigeon breeder's lung compared with those with usual interstitial pneumonia. Am Rev Respir Dis. 1993;148(1):49-53.

7. Sahin H, Brown KK, Curran-Everett D, et al. Chronic hypersensitivity pneumonitis: CT features comparison with pathologic evidence of fibrosis and survival. Radiology. 2007;244(2):591-598.

8. Vourlekis JS, Schwarz MI, Cherniack RM, et al. The effect of pulmonary fibrosis on survival in patients with hypersensitivity pneumonitis. Am J Med. 2004;116(10): 662-668.

9. Walsh SL, Sverzellati N, Devaraj A, Wells AU, Hansell DM. Chronic hypersensitivity pneumonitis: high resolution computed tomography patterns and pulmonary function indices as prognostic determinants. Eur Radiol. 2012;22(8):1672-1679.

10. Wang $\mathrm{P}$, Jones KD, Urisman A, et al. Pathologic findings and prognosis in a large prospective cohort of chronic hypersensitivity pneumonitis. Chest. 2017;152(3):502-509.

11. Raghu G, Collard HR, Egan JJ, et al. An official ATS/ERS/JRS/ALAT statement: idiopathic pulmonary fibrosis: evidencebased guidelines for diagnosis and management. Am J Respir Crit Care Med. 2011;183(6):788-824.

12. Chiba S, Tsuchiya K, Akashi T, et al. Chronic hypersensitivity pneumonitis with a usual interstitial pneumonia-like pattern: correlation between histopathologic and clinical findings. Chest. 2016;149(6):1473-1481.

13. Churg A, Muller NL, Flint J, Wright JL. Chronic hypersensitivity pneumonitis. Am J Surg Pathol. 2006;30(2):201-208.

14. Lynch DA, Newell JD, Logan PM, King TE Jr, Muller NL. Can CT distinguish hypersensitivity pneumonitis from idiopathic pulmonary fibrosis? AJR Am J Roentgenol. 1995;165(4):807-811.

15. Silva CI, Muller NL, Lynch DA, et al. Chronic hypersensitivity pneumonitis: differentiation from idiopathic pulmonary fibrosis and nonspecific interstitial pneumonia by using thin-section CT. Radiology. 2008;246(1):288-297.

16. Flaherty KR, King TE Jr, Raghu G, et al. Idiopathic interstitial pneumonia: What is the effect of a multidisciplinary approach to diagnosis? Am J Respir Crit Care Med. 2004;170(8):904-910.

17. Society AT. Idiopathic pulmonary fibrosis: diagnosis and treatment. International consensus statement. American Thoracic Society (ATS), and the European Respiratory Society (ERS). Am J Respir Crit Care Med. 2000;161(2 Pt 1):646-664.

18. Macintyre N, Crapo RO, Viegi G, et al. Standardisation of the single-breath determination of carbon monoxide uptake in the lung. Eur Respir J. 2005;26(4):720735.

19. Miller MR, Hankinson J, Brusasco V, et al. Standardisation of spirometry. Eur Respir J. 2005;26(2):319-338.

20. Wanger J, Clausen JL, Coates A, et al. Standardisation of the measurement of lung volumes. Eur Respir J. 2005;26(3): 511-522.

21. Salisbury MG, Chughtai A, Sayyouh M, et al. Hypersensitivity pneumonitis and idiopathic pulmonary fibrosis patients with similar radiologic phenotype experience similar outcomes. Presented at: Pulmonary Fibrosis Foundation Summit; November 9-11, 2017; Nashville, TN.

22. Salisbury ML, Gross BH, Chughtai A, et al. Development and validation of a radiologic diagnosis model for 
hypersensitivity pneumonitis. Eur Respir I. 2018;52(2)

23. Hansell DM, Bankier AA, MacMahon $H$, McLoud TC, Muller NL, Remy J. Fleischner Society: glossary of terms for thoracic imaging. Radiology. 2008;246(3):697-722.

24. Harrell FE Jr, Lee KL, Mark DB. Multivariable prognostic models: issues in developing models, evaluating assumptions and adequacy, and measuring and reducing errors. Stat $\mathrm{Med}$. 1996;15(4):361-387.

25. Cox DR. Regression models and life tables (with discussion). J R Stat Soc Series B Stat Methodol. 1972;34:187-220.

26. Chung JH, Zhan X, Cao M, et al. Presence of air trapping and mosaic attenuation on chest computed tomography predicts survival in chronic hypersensitivity pneumonitis. Ann Am Thorac Soc. 2017;14(10):1533-1538.
27. Jacob J, Bartholmai BJ, Egashira R, et al. Chronic hypersensitivity pneumonitis: identification of key prognostic determinants using automated CT analysis. BMC Pulm Med. 2017;17(1):81.

28. Oldham JM, Adegunsoye A, Valenzi E et al. Characterisation of patients with interstitial pneumonia with autoimmune features. Eur Respir J. 2016;47(6):17671775.

29. Kim EJ, Elicker BM, Maldonado F, et al. Usual interstitial pneumonia in rheumatoid arthritis-associated interstitial lung disease. Eur Respir J. 2010;35(6): 1322-1328.

30. Lacasse Y, Selman M, Costabel U, et al. Classification of hypersensitivity pneumonitis: a hypothesis. Int Arch Allergy Immunol. 2009;149(2): 161-166.
31. Richerson HB, Bernstein IL, Fink JN, et al. Guidelines for the clinical evaluation of hypersensitivity pneumonitis. Report of the Subcommittee on Hypersensitivity Pneumonitis. J Allergy Clin Immunol. 1989;84(5 Pt 2):839-844.

32. Vasakova M, Morell F, Walsh S, Leslie K, Raghu G. Hypersensitivity pneumonitis: perspectives in diagnosis and management. Am J Respir Crit Care Med. 2017;196(6):680-689.

33. Lima MS, Coletta EN, Ferreira RG, et al. Subacute and chronic hypersensitivity pneumonitis: histopathological patterns and survival. Respir Med. 2009;103(4):508-515.

34. Fernandez Perez ER, Swigris JJ, Forssen AV, et al. Identifying an inciting antigen is associated with improved survival in patients with chronic hypersensitivity pneumonitis. Chest. 2013;144(5):1644-1651. 